Preprint

UCRL-JC-135153

\title{
Development of Adaptive Resonator Techniques for High-Power Lasers
}

\author{
L. Flath, J. An, J. Brase, C. Carrano, C.B. Dane, S. Fochs, \\ R. Hurd, M. Kartz, R. Sawvel
}

This article was submitted to International Workshop on Adaptive Optics for Industry and Medicine, Durham, England, July 12-16, 1999

\section{July 12, 1999}

U.S. Department of Energy

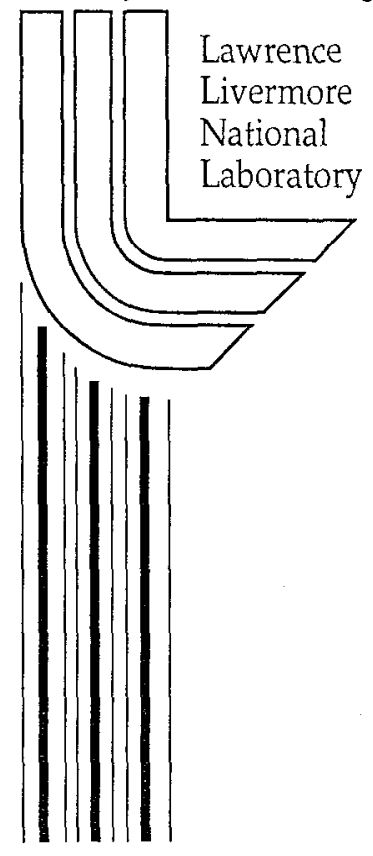




\section{DISCLAIMER}

This document was prepared as an account of work sponsored by an agency of the United States Government. Neither the United States Government nor the University of California nor any of their employees, makes any warranty, express or implied, or assumes any legal liability or responsibility for the accuracy, completeness, or usefulness of any information, apparatus, product, or process

disclosed, or represents that its use would not infringe privately owned rights. Reference herein to any specific commercial product, process, or service by trade name, trademark, manufacturer, or otherwise, does not necessarily constitute or imply its endorsement, recommendation, or favoring by the United States Government or the University of California. The views and opinions of authors expressed herein do not necessarily state or reflect those of the United States Government or the University of California, and shall not be used for advertising or product endorsement purposes. 


\title{
DEVELOPMENT OF ADAPTIVE RESONATOR TECHNIQUES FOR HIGH-POWER I,ASERS
}

\author{
LAURENCE FLAT'H, JONG AN, JAMES BRASE, CARMEN CARRANO, C. BRENT \\ DANE, SCOTT FOCHS, RANDALL HURD, MICHAEL KARTZ, AND ROBERT SAWVEL \\ Lawrence Livermore National Laboratory, P.O. Box 808, L-258, Livermore, CA 94551, USA \\ E-mail: flathI@llnl.gov
}

\begin{abstract}
The design of an adaptive wavefront control system for a high-power Nd:Glass laser will be presented. Features of this system include: an unstable resonator in confocal configuration, a multi-module slab amplifier, and real-time intracavity adaptive phase control using deformable mirrors and high-speed wavefront sensors. Experimental results demonstrate the adaptive correction of an aberrated passive resonator (no gain).
\end{abstract}

\section{Introduction}

Solid-state amplifiers present obstacles to the objective of high average power lasers; they provide a low gain per pass and suffer from wavefront distortions due to thermal gradients in the lasing material. The low amplifier gain requires a low magnification resonator, which increases the sensitivity of wavefront quality to aberrations and alignment errors [1]. When operated in a heat-capacity mode [2], the thermal aberrations are time-varying, thus preventing the use of a static corrector optic.

An adaptive optics system provides a means to control the wavefront evolution in laser cavities [3-10]. Intracavity wavefront sensing allows real-time adaptive control of the resonator's mode structure and vastly improves the output beam quality. In this paper we present the successful implementation of high-spatial resolution intracavity adaptive phase control in a confocal unstable resonator with a sub-threshold Nd:Glass disk amplifier. This work lays the foundation for extentions to high average power heat-capacity laser systems.

\section{Adaptive Unstable Resonators}

Using intracavity wavefront correction in unstable resonators has several advantages compared to extracavity sensing [8-10]. Using a high-spatial resolution wavefront sensor and adaptive optics (Figure 1), it is possible to directly control the mode shape. Alignment sensitivity is reduced because individual components of the wavefront (e.g. tip/tilt) may be extracted and acted upon by dedicated active components. And because the field propagated through multiple passes in the cavity, the corrector's dynamic range requirements are reduced.

A099 Paper.doc submitted to World Scientific : 07/20/99 : 1:15 PM 


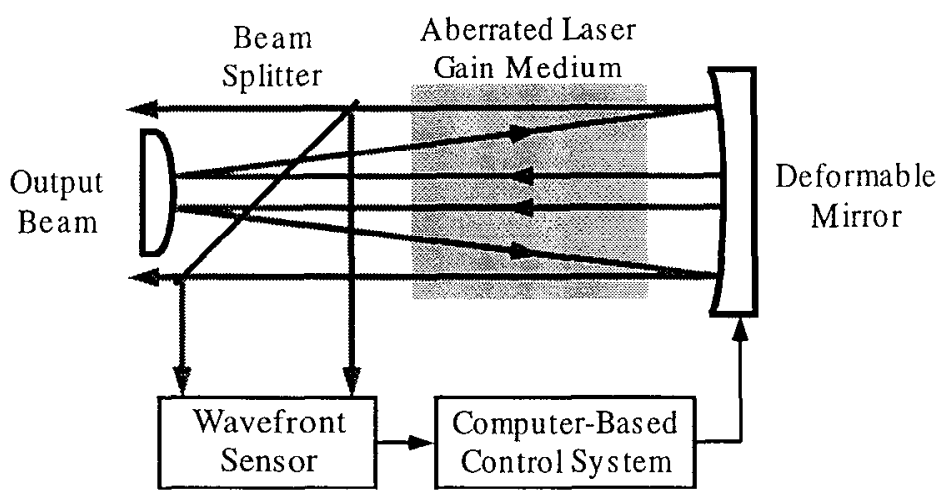

Figure 1. Adaptive wavefront correction in a positive-branch unstable resonator.

Of course, there are also difficulties with this type of arrangement. Aberrations in the laser medium are magnified in each round-trip pass through the cavity, and thus lower the output wavefront quality and far-field Strehl ratio. The correction calculations are more complex than a simple phase mapping; the aberration mode amplification is dependent on mode order and cavity position [10].

Intracavity control of an unstable resonator was first demonstrated to correct misalignments in a $\mathrm{CO}_{2}$ laser by Stephens and Lind [9]. In these and subsequent experiments, the deformable mirror was controlled using a multi-dither approach based on feedback from the far-field peak intensity $[6,7]$. In the work described in this paper, we use high order intracavity wavefront sensing to measure the spatially resolved field at the laser output. The full wavefront information is then used to directly control the deformable mirror. The experiments described here demonstrate correction of an aberrated passive resonator (no gain) - future experiments will use the system to correct an amplificd beam. The experiment consists of a positive branch unstable resonator with magnification $M=1.31$ and equivalent Fresnel number $N_{e y}=20.4$. The aberration is provided by a prototype flashlamp-pumped Nd:Glass heat capacity amplifier, which can be pumped to a temperature which provides approximately 2.0 waves of low-order aberration in one pass. In the experiment, the amplifier is heated by flashlamp pumping, then the adaptive resonator control loop is closed to correct an injected probe beam.

The heart of our system is a negative feedback loop that adaptively controls the surface of a deformable mirror (DM), using difference between the time-varying wavefront gradient and the desired wavefront slope (flat) to form an error signal. The relation between the error signal and the surface profile of the DM is described by a control law:

$$
\vec{\beta}=\mathbf{S} \vec{\alpha} \rightarrow \vec{\alpha}=\mathbf{A} \vec{\beta}
$$


where $\vec{\alpha}$ and $\vec{\beta}$ are vectors describing the intra-cavity wavefront slope and the surface function of the DM, respectively. $S$ is a matrix describing the feedback system. The matrix $\mathbf{S}$ is calculated as the pseudoinverse of the system matrix $\mathbf{A}$ [11].

The systcm matrix is measured by a calibration procedure where the influence of each individual DM actuator on the wavefront slope is recorded, the combined result (the forward matrix $\mathbf{A}$ ) is inverted using singular-value decomposition [12], and the eigenmodes of the system are weighted according to the desired system response. In this manner modes by which the DM has no or little influence may be rejected, analogous to the action of a spatial filter. Note that for an intracavity DM, the wavefront sensor response is more complex than in a single-pass system. For the passive case described here, the wavefront sensor measures the weighted sum of multiple passes through the resonator.

\section{Passive Resonator Testbed System}

As a first step toward a high-average power laser, we constructed a testbed system, with the objective of verifying the general approach for wavefront sensing and control. The experimental setup (schematic shown in Figure 2) consists of a probe laser, an unstable resonant cavity (URC), a deformable mirror (DM), a solid-state laser amplifier module, a wavefront sensor (WFS), and a far-field sensor (FFS).

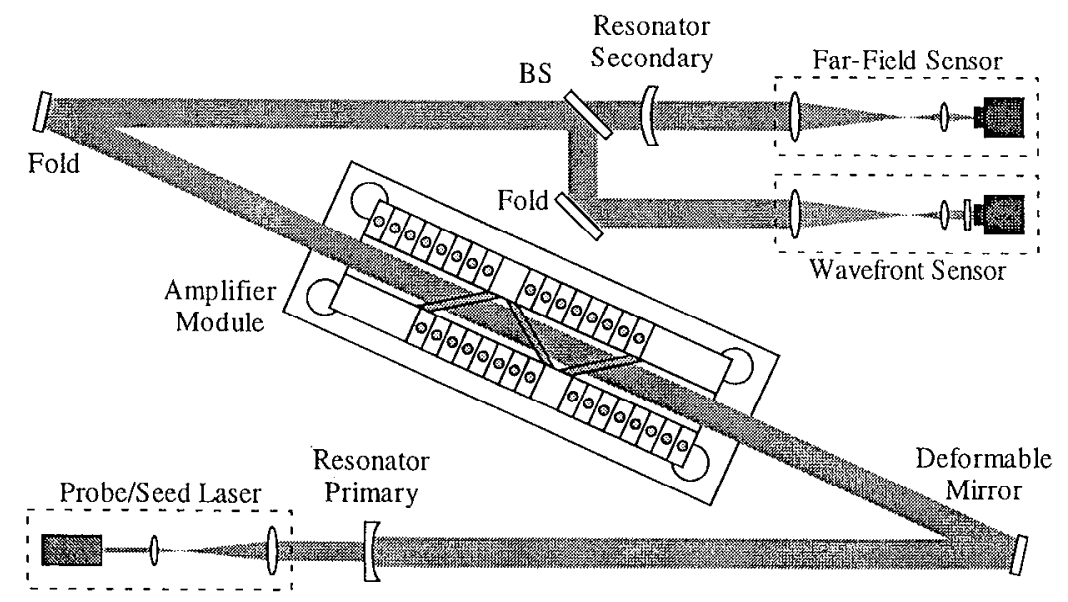

Figure 2. Schematic layout of the adaptive unstable passive resonator testbed.

The URC consists of a $97 \%$ reflective primary of radius $39.5 \mathrm{~m}$, and a $-30.1 \mathrm{~m}$ radius secondary mirror with a $51 \mathrm{~mm}$ diameter $97 \%$ reflective central coating. The geometry of the secondary optic couples the laser output energy into an annular

A099 Paper.doc submitted to World Scientific : 07/20/99 : 1:15 PM 
cross-section. The mirrors are aligned confocally, resulting in a cavity length of $4.7 \mathrm{~m}$ and a resonator magnification $M=1.31$. The URC is seeded with a solidstate probe-laser emitting CW radiation at $1053 \mathrm{~nm}$, and is expanded to a diameter of $51 \mathrm{~mm}$ to match the central reflective zone of the secondary mirror. The beam is injected through the primary mirror and collimated at the cavity-side of the primary. Thus, one round-trip pass of the probe beam results in an output annulus outer diameter of $M \cdot 51 \mathrm{~mm}=67 \mathrm{~mm}$. The deformable mirror has 109 P7T actuators in a hexagonal-close-pack arrangement, with spacing of $7 \mathrm{~mm}$ and stroke of $\pm 2.7 \mu \mathrm{m}$, and a surface roughness of $19 \mathrm{~nm}$ RMS (165 nm P-V, mostly edges). The laser amplifier consists of three flashlamp-pumped Nd:Glass slabs alternately oriented at Brewster's angle of $56.6^{\circ}$. For the current pump/resonator configuration, the laser runs just below threshold. During operation the amplifier is pumped at a rate of $10 \mathrm{~Hz}$ for 15 seconds. The intracavity wavefront is sampled at the plane of the DM by a Shack-Hartmann sensor consisting of a reducing telescope and a lenslet array (750 $\mu \mathrm{m}$-spaced lenslets, $f=20.1 \mathrm{~mm}$ ). The spot pattern is imaged directly onto the focal plane of a 12-bit digital camera, which uses a $1024 \times 1024$ pixel frame transfer CCD. Images are acquired at the camera's full frame rate of $8.4 \mathrm{~Hz}$. A far field sensor consisting of a magnifying telescope and an identical digital camera are used to monitor the annular output of the cavity. Figure 3 shows the relationship between the beam profile, the DM actuator positions, and the wavefront sampling of the WFS.

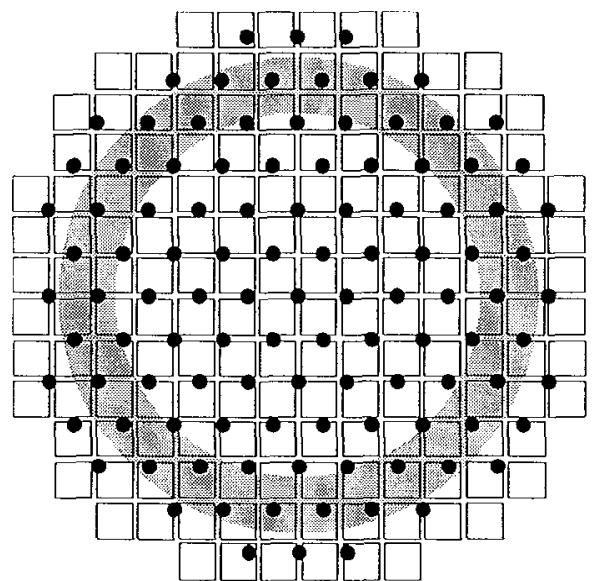

Figure 3. Relative position of deformable mirror actuators (dots) and wavefront sensor lenslets (squares) with respect to the output beam (annulus).

The system controller is a UNIX workstation with two digital camera inputs and the DM output interface. Control loop operation consists of determining the wavefront slopes from the spot positions on the WFS camera, multiplying by the system matrix $\mathbf{S}$, and sending the result to the DM. 


\section{Experimental Results}

The results of closed-loop operation of the adaptive optical system are shown in Figure 4 . The system was calibrated by measuring the intracavity wavefront before pumping the amplifier, yielding a control loop reference wavefront. To generate the worst-case aberrations, the Nd:Glass slabs were heated by pulsing the laser amplifier flashlamps 150 times. The uncorrected far-field image in Figure 4a has a residual wavefront error of $1.96 \lambda \mathrm{rms}$, and a calculated Strehl ratio of 0.04 . With this as the starting point for the controller, the output in Figure $4 \mathrm{~b}$ resulted after 19 control loop iterations - a residual wavefront error of $0.16 \lambda \mathrm{rms}$, and a Strehl ratio of 0.31 (an eight-fold improvement).

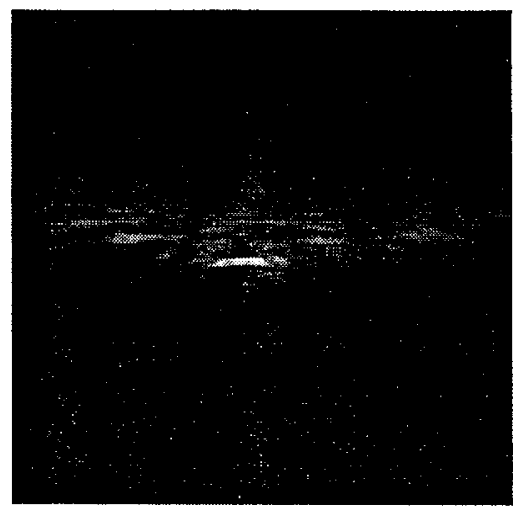

(a)

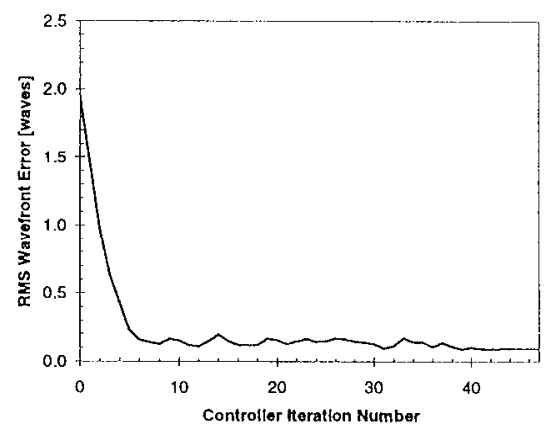

(c)

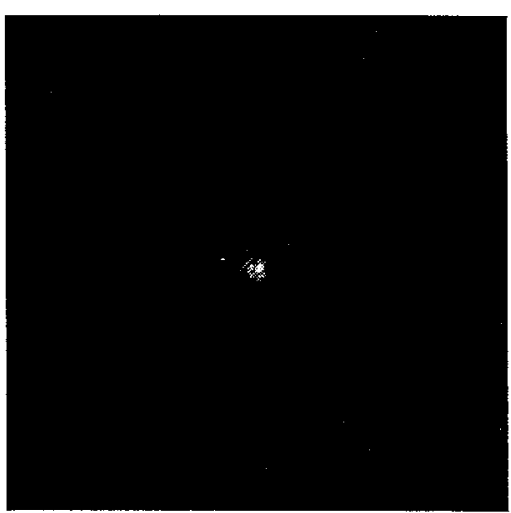

(b)

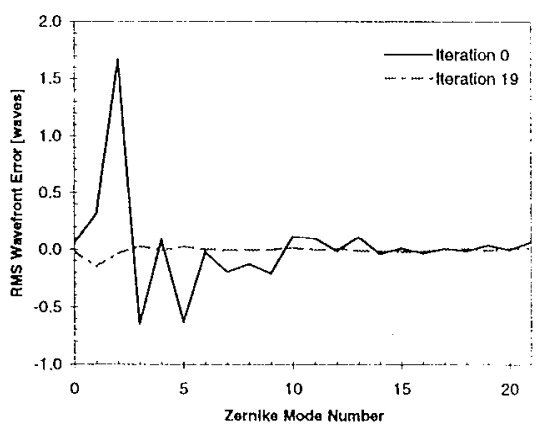

(d)

Figure 4. Testbed system far-field images (a) immediately after 150 pulses induce intracavity aberrations, and (b) after nineteen iterations of adaptive loop control. Controller performance (c) over time in terms of control loop iteration number, and (d) Zernike mode decomposition of the wavefront error. 
To evaluate the performance of the controller we decompose the wavefront into its constituent Zernike modes (Figure 4c). It is clear that most of the initial wavefront error is of low order, as we expect for this type of laser amplifier [1]. Figure 4d shows that the algorithm converges in less than 10 iterations.

\section{Acknowledgements}

The authors would like to acknowledge the support and technical assistance of G. F. Albrecht, B. S. Bhachu, B. J. Bauman, D. T. Gavel, L. G. Lindberg, W. J. Manning, E. A. Stappaerts, and J. D. Wintemute. This research was funded by the U.S. Army High-Energy Laser Test Facility under WFO Proposal No. L7378.

\section{References}

1. Koechner W. Solid-State Laser Engineering, Fourth Edition, (Springer-Verlag, Berlin, 1996) pp. 262-278.

2. Albrecht G. F., Sutton S. B., George E. V., Sooy W. R., and Krupke W. F. Solid state heat capacity disk laser, Laser and Particle Beams 16-4 (1998) pp. 605-625.

3. Siegman A. E. Unstable optical resonators, Applied Optics 13-2 (1974) pp. 353367.

4. Freeman R. H., Freiberg R. J., and Garcia H. R. Adaptive laser resonator, Optics Letters 2-3 (1978) pp. 61-63.

5. Oughstun K. E. Intracavity adaptive optic compensation of phase aberrations. I: Analysis, Journal of the OSA 71-7 (1981) pp. 862-872.

6. Spinhirne J. M., Anafi D., Freeman R. H., and Garcia H. R. Intracavity adaptive optics. I. Astigmatism correction performance. Applied Optics 20-6 (1981) pp. 976-984.

7. Anafi D., Spinhirne J. M., Freeman R. H., and Oughstun K. E. Intracavity adaptive optics. II. Tilt correction performance. Applied Optics 20-11 (1981) pp. 1926-1932

8. Kudryashov A. V. Intracavity laser beam control, Proceedings of the SPIE 3611 (1999) pp. 32-41.

9. Stephens R. R. and Lind R. C. Experimental study of an adaptive-laser resonator, Optics Letters 3-3 (1978) pp. 79-81.

10. Oughstun K. E. Theory of intracavity adaptive optic mode control, Proceedings of the SPIE 365 (1982) pp. 54-65.

11. Tyson R. K. Principles of Adaptive Optics, Second Edition, (Academic Press, San Diego, 1998).

12. Press W. H., Teukolsky S. A., Vetterling W. T., and Flannery B. P. Numerical Recipes in C: The Art of Scientific Computing, Second Ed. (Cambridge University Press, Cambridge, 1992) pp. 59-70.

This work was performed under the auspices of the U.S. Department of Energy by Lawrence Livermore National Laboratory under contract No. W-7405-Eng-48. 